\title{
THE MANAGEMENT OF CARE IN CARDIOVASCULAR NURSING
}

\author{
Marluci Andrade Conceição Stipp
}

The genesis of cardiovascular diseases is multifactorial, as it involves several elements, of which I highlight: the epidemiological profile, living with the risk factors for the development of cardiovascular diseases, the complex determination of health-disease process with several possibilities of expression of our genome in relation to the environment.

Several publications show that living with risk factors increases with age, and the association between them adds significantly the risk of morbi-mortality. Within the epidemiological concept, the cardiovascular diseases are included in the group of non-contagious chronic diseases or even, in chronic health conditions which are characterized by the absence of microorganisms, for non-transferability, irreversibility and multiplicity of risk factors.

The Brazilian research has grown increasingly in the area of cardiology, and nursing has contributed effectively to this growth. The understanding of nurses in how the problem of cardiovascular disease is part of the everyday care, will allow an application of healthy care methodologies in preventing these diseases in order to facilitate the administration of nursing assistance. This is the way too, that will be provided a creative care, human, engaged in all factors that favor the action of self-care.

Despite all the development of therapeutic strategies, based especially on interventionist actions, prevention is what brings back the care as the best way to follow. In the administration of nursing care when using the assistance models, in particular the management model of the health-disease process, that will be closer to the customer, from his environment, considering the health-disease process and everything around. It is a continuous search looking for new strategies to face the disease, to promote the well-being and quality of health.

We emphasize that the main cause of these diseases is not the genetic but environmental and behavioral risk factors that can be modified. New and different approaches that arise to health promotion in nursing, offer a new perspective to reaffirm its importance in our profession, especially applying a strategic view to realize the care in a preventive way and with a greater coverage.

Based on this vision we need to postulate a cardiovascular nursing care also based on a larger approach, to propose a care methodology considering the vision of taking human beings as unique, free for their choices, but aware of the existence of their problems and their serious consequences. 